\title{
Consentimiento informado: algunas consideraciones actuales
}

Roberto Cañete ${ }^{1}$, Dirce Guilhem² ${ }^{2}$ Katia Brito ${ }^{3}$

Resumen: El consentimiento informado constituye un eslabón crucial en las investigaciones que involucran seres humanos. Sin embargo, limitaciones del conocimiento que conducen a errores en su confección y/o aplicación han sido frecuentemente identificadas entre los profesionales a escala global. En correspondencia, se expone una breve revisión temática de su concepto y de los elementos relacionados con su adecuada confección y aplicación, lo que, creemos, permitirá clarificar la información que poseen los profesionales que conducen investigaciones con seres humanos.

Palabras clave: bioética, consentimiento informado, fundamentos éticos

\section{Informed consent: some current considerations}

Abstract: Informed consent constitutes a crucial link in research involving human beings. Nevertheless, knowledge limitations which lead to errors of its process and application have been frequently identified by professionals globally. Hence, a brief review of the topic is exposed about the concept and the elements related to adequate process and application, which we belief will allow to clarify the information that professionals who do research involving human beings posses.

Key words: bioethics, informed consent, ethics foundations

\section{Consentimento informado: algumas consideraçóes atuais}

Resumo: $\mathrm{O}$ consentimento informado constitui um obstáculo crucial nas pesquisas que envolvem seres humanos. No entanto, limitaçóes do conhecimento que conduzem a erros em sua confecção e/ou aplicação têm sido frequentemente identificadas entre os profissionais em escala global. Em correspondência, se expóe uma breve revisão temática de seu conceito $\mathrm{e}$ dos elementos relacionados com a sua adequada confecçáo e aplicaçáo, o que cremos permitirá esclarecer a informação que possuem os profissionais que conduzem pesquisas com seres humanos.

Palavras-chave: bioética, consentimento informado, fundamentos éticos

\footnotetext{
${ }^{1}$ Doctor en Ciencias Médicas. Centro Provincial de Higiene, Epidemiología y Microbiología, Matanzas, Cuba Correspondencia: roberto.villafranca@infomed.sld.cu

${ }^{2}$ Doctora en Bioética. Profesora Titular de la Universidad de Brasilia, Brasilia, Brasil

${ }^{3}$ Doctora en Estomatología. Profesora e Investigadora, Policlínica Samuel Fernández, Matanzas, Cuba
} 


\section{Introducción}

Debido al impacto creciente de los avances de la ciencia y la tecnología en el mundo actual, así como la influencia de la investigación científica en la creación de soluciones a los problemas de la vida, los aspectos éticos han emergido con inigualable fuerza en el debate internacional(1). Ello demanda la responsabilidad de la comunidad científica, ya que la credibilidad, la integridad y los motivos por los cuales se conducen las investigaciones se evalúan en escala social y los resultados de esas investigaciones se incorporan, en mayor o menor grado, al estilo de vida de los seres humanos dondequiera que habiten(2).

Los servicios sanitarios también han evolucionado y el mayor reflejo, al menos en su modo de actuar, se evidencia en la relación médico-paciente: la relación entre un individuo con necesidades de salud y otro u otros con herramientas y conocimientos para satisfacer esas necesidades. La relación que en un pasado cercano era eminentemente paternalista (medicina centrada en el médico) se ha transformado en una más participativa (medicina centrada en el paciente), en la cual cada individuo exige conocer todo lo relacionado con su persona(3).

Diferentes autores han expuesto, sin embargo, su inconformidad con la dimensión conceptual de "la relación médico-paciente" por considerarla poco precisa $(4,5)$. Para respaldar sus planteamientos exponen que el médico no es el único que brinda atención a la salud de los individuos, también lo hace la enfermera, el odontólogo, el psicólogo clínico, etc., cuya relación con el individuo es tan intersubjetiva y humana como la de aquél. Por otro lado, tampoco es necesariamente un paciente el que solicita este tipo de atención. En muchos casos el objetivo de la consulta es determinar si se está o no enfermo, por lo tanto, los sanos también intervienen en esta clase de relación.

Lo anterior conduce a la necesidad de considerar que la denominación "médico-paciente" debe ser sustituida por una de carácter genérico, como por ejemplo relación equipo de salud-usuario, en la que todos los profesionales de la atención a la salud tienen las mismas responsabilidades ante los solicitantes, independientemente de su capacitación y formación profesional específica.

Existen principios éticos que rigen el quehacer de los profesionales de la salud y de los investigadores en general, principios universales e históricos que conservan total vigencia y que deberían aplicarse en todo momento y ser de obligatorio cumplimiento para los profesionales de la salud pública. Dentro de esos principios comúnmente se exponen: el respeto a la vida, la autonomía, la beneficencia y no maleficencia, y la justicia(6).

En ese marco, el consentimiento informado (CI) se convierte en una herramienta de mediación entre los intereses de investigadores, financiadores e individuos incluidos en la investigación científica. El CI es, adicionalmente, una excelente forma de ejercitar y aplicar principios éticos tan genuinamente humanos y universales. También conocido como consentimiento libre y esclarecido, consentimiento bajo información o consentimiento educado, el CI permite garantizar la autonomía, la autodeterminación y el respeto a los individuos involucrados en el proceso de investigación científica o que reciben atención médica.

Contrario a lo que se pudiera imaginar, la aplicación de los más elementales principios éticos encuentra enormes dificultades en la práctica médica internacional. Un estudio publicado en fecha reciente en Pakistán(7) evidenció que la adherencia a principios éticos era inadecuada y que existía la imperiosa necesidad de incorporar esos temas en los programas educativos de pre y posgrado en ese país. Esa misma investigación identificó claras limitaciones en la práctica del CI en dos hospitales de atención terciaria. Otros estudios reflejan las mismas limitaciones(8-10).

En concordancia con lo expresado y como respuesta a las necesidades cognitivas de los profesionales de la salud y de investigadores en general, se expone el concepto y los fundamentos éticos del consentimiento informado, identificando, adicionalmente, sus límites y las "reglas de oro" al momento de confeccionar este complemento imprescindible del proceso de investigación científica. 


\section{La vía para un concepto}

El término "bioética" fue utilizado por Van Rensselaer Potter para referirse a los problemas surgidos a partir del desarrollo vertiginoso de la ciencia y la tecnología en un mundo imbuido en una profunda crisis de valores, y a la necesidad de superar la ruptura entre investigación científica y humanidad(11). Esta ruptura se refleja en la asimetría existente entre el enorme desarrollo tecnológico actual, que otorga al hombre el poder de manipular la intimidad del ser humano y de alterar el medio, y la ausencia de un aumento correlativo en su sentido de responsabilidad. El vertiginoso desarrollo científico-técnico es muy superior al alcanzado por el discurso ético.

En ese marco, surge el CI con el propósito de asegurar la decisión autónoma de la persona de participar o no en una investigación según esta se ajuste o no a sus valores, intereses y preferen$\operatorname{cias}(12)$.

En el proceso de obtención del CI se debe ofrecer información clara y precisa sobre el objetivo de la investigación, los riesgos y beneficios de la misma, así como las alternativas posibles. Igualmente, se debe informar a los individuos que su participación es voluntaria, que pueden retirarse libremente cuando lo juzguen conveniente, que se guardará confidencialidad de sus datos, que los daños resultantes de la investigación les serán resarcidos y que su negativa a participar en el estudio no afectará sus derechos como paciente(13).

El primer código que pretendía establecer criterios de regulación para la investigación en seres humanos se redactó, paradójicamente, en Alemania en 1931 $(14,15)$. Sin embargo, correspondió al Código de Nürenberg, surgido a partir de los actos contra la humanidad cometidos por los nazis, ser uno de los primeros documentos que pretendió ser norma internacional para la protección de los sujetos que participan en investigación.

El Código de Nürenberg, de 1947, fue el primer código, luego de la regulación alemana antes mencionada, que plantea el derecho del individuo a dar su "consentimiento voluntario" y que especifica la importancia de esta regla en tanto sostiene en su artículo primero que: "El consentimiento voluntario del sujeto humano es absolutamente esencial", especificando además que el sujeto debe gozar de capacidad legal y competencia para realizar una elección libre y estar completa y verazmente informado para llevarla a cabo(16).

A pesar de su importancia, el Código tuvo escasa repercusión en los ámbitos médicos y científicos de la época, ya que "era un código para los médicos bárbaros pero no para los médicos americanos"(16). En 1964 estos conceptos fueron ampliados en la Declaración de Helsinki, en las llamadas "recomendaciones para guiar la investigación en seres humanos", y más tarde en el Informe Belmont, de 1978, y otras declaraciones similares(17).

\section{Concepto}

El colegio americano de médicos definió el CI como "la explicación a un paciente atento y mentalmente competente, de la naturaleza de su enfermedad, así como el balance de los efectos de la misma y el riesgo de los procedimientos diagnósticos y terapéuticos recomendados, para a continuación solicitarle su aprobación para ser sometido a esos procedimientos". Esa misma instancia agrega: "La presentación de la información debe ser comprensible y no sesgada (...), la colaboración del paciente debe ser conseguida sin coacción y (...) el médico no debe sacar partido de su potencial dominio psicológico sobre el paciente" (18).

Es importante mencionar que el CI inadecuado carece de valor legal y/o moral y, aun siendo adecuado, la firma del mismo no exime al médico de responsabilidad en caso de negligencia. El médico que no informe será responsable de los daños provocados por los procedimientos que practique, aunque no exista mala práctica profesional, de ahí que el CI puede perder su función protectora en el ámbito médico-legal si no se realiza de forma correcta. Por otro lado, no hay que olvidar que la mayor protección frente a litigios consiste en una buena comunicación con los pacientes. 
El contenido del CI y el procedimiento mediante el que se consigue están fuertemente determinados por el lenguaje. La mala comunicación y/o entendimiento acerca de los elementos constitutivos del CI son más frecuentes si el investigador y el sujeto en investigación hablan idiomas diferentes o el documento debe ser traducido. Por ese motivo, los documentos internacionales enfatizan en la necesidad de clarificar y simplificar el lenguaje utilizado en el documento escrito y en la información que se brinda oralmente a los suje$\operatorname{tos}(19)$.

El CI describe un proceso interactivo en el cual el individuo (o su representante legal) accede voluntariamente y sin coerción a participar en un estudio, luego de que los propósitos, riesgos y beneficios de este han sido cuidadosamente expuestos y entendidos por las partes involucradas. En consecuencia, el CI no solo es necesario e imprescindible en una investigación científica, sino que tiene igual significación en cualquier momento en que el individuo necesite, por razones diversas, alguna intervención médica.

El CI es tan importante y universalmente aceptado que diferentes organizaciones sociales y entidades reguladoras expresan su inquietud en relación con el derecho del paciente a influir "de alguna manera" en las decisiones médicas, con lo cual esperan se garantice el respeto a su autonomía, independientemente de la situación en que el paciente se encuentre(20).

\section{Consentimiento informado y grupos vulnera- bles}

Los profesionales que conducen investigaciones que involucran seres humanos deben tener especial precaución con los individuos vulnerables, es decir, los que tienen acentuada incapacidad de proteger sus propios intereses(21). Vulnerables son aquellos individuos, comunidades o sectores de la comunidad expuestos a recibir daño o abuso debido a que su autonomía se encuentra menoscabada o disminuida $(22,23)$.

En términos generales, se acepta que la vulnera- bilidad puede ser motivada por alguna(s) de las siguientes condiciones:

- Ser menor de edad.

- Tener una grave discapacidad sensorial que dificulte la comprensión.

- No dominar el idioma con fluidez.

- Padecer algún trastorno psiquiátrico agudo o crónico.

- Padecer un grave deterioro cognitivo.

- Reclusos o personas en instituciones en las que por motivos jerárquicos terceros pudieran ejercer influencias coercitivas.

- Pobreza.

Las personas que cumplan alguna(s) de estas condiciones podrían ser catalogadas como "vulnerables en extremo" y, en tal caso, los investigadores deberían generar estrategias especiales que garanticen la salvaguarda de sus derechos.

\section{Identificando los límites del consentimiento informado}

Los individuos sujetos de investigación o sus representantes legales, independientemente de su condición, deben tener pleno conocimiento de lo relacionado con su salud, a no ser que expresen formalmente lo contrario. Es así como se identifican los llamados "límites del consentimiento informado" que de manera resumida se exponen a continuación:

- El paciente tiene derecho a no ser informado si así lo expresa previamente.

- En caso de riesgo para la salud pública, se acepta la no existencia de consentimiento informado para el internamiento, cuarentena $\mathrm{u}$ hospitalización del paciente.

- En caso de riesgo inmediato grave para la integridad física o psíquica del paciente, el consentimiento puede obviarse.

- En caso de pacientes menores de edad o de incapacidad legalmente reconocida, física o mental, se admite que el consentimiento informado sea pedido a su representante legal, que será generalmente el familiar más próximo.

- En caso de riesgo grave para la salud pública o la vida del paciente, el consentimiento del 
representante legal solo se tendrá en cuenta.

- El paciente puede revocar libremente, por escrito, su consentimiento en cualquier momento.

\section{Lo que caracteriza al consentimiento informa- do}

Bedrossian y Fernández(24), en un artículo publicado en 2001, se refieren a algunas de las características más importantes del CI. Estas características sintetizan en buena medida las generalidades éticas del CI por lo que se prefiere exponerlas íntegramente.

\section{Según esos autores:}

- El consentimiento informado es un proceso de comunicación entre el profesional de la salud y el usuario, que culmina con la autorización o no de una intervención clínica específica.

- El consentimiento es un derecho del paciente; proporcionar la información es un deber del médico.

- La información y la comprensión son las únicas herramientas decisivas para dar el consentimiento.

- La información debe especificar los riesgos de un procedimiento en orden de su frecuencia y gravedad; no debe ser la síntesis de un tratado de patología.

- La información debe adecuarse a las condiciones particulares de cada paciente, familiares o representantes.

- En el desarrollo de una atención médica pueden requerirse nuevos y sucesivos consentimientos.

- El usuario tiene derecho a revocar su consentimiento en cualquier etapa del desarrollo de la relación clínica, sin que ello conlleve al detrimento en la calidad de su atención en salud.

- Idealmente, el consentimiento puede otorgarse después de contar con varios días de reflexión.

- El consentimiento es una parte sustantiva de la atención médica y no un mero acto administrativo a cargo de personal no médico.
- El responsable del consentimiento informado es el profesional de la salud directamente comprometido con la atención del paciente.

- La mejor prueba del acto del consentimiento es lo escrito y firmado en la historia clínica.

- El consentimiento no es válido cuando, por el estado de salud o la acción de medicamentos, el paciente no está en condiciones de elaborar un juicio crítico; en estos casos es importante el consentimiento de sus familiares o responsables.

- Si se trata de incapaces declarados por ley (menores de edad, discapacitados mentales, etc.), es necesario contar con el consentimiento de sus familiares o responsables.

- Las necropsias, salvo las ordenadas legalmente, deben ser consentidas; la autorización o negativa debe registrarse en la historia clínica, que constituye la prueba documental por excelencia.

- La ausencia del consentimiento debilita la defensa profesional en actos legales.

- El consentimiento no es una dispensa de culpa. No sirve para eludir la responsabilidad profesional.

- Es importante detallar en la historia clínica qué circunstancias obligaron a omitir el consentimiento.

- La atención médica actual, fraccionada, sucesiva y múltiple requiere de la organización institucional el deber de informar y advertir.

Adicionalmente, en la confección del CI los investigadores deben cumplir tres condiciones o reglas de oro: proveer información suficiente y de calidad, adecuar la información al nivel de quien la recibirá y procurar la voluntariedad de participación sin coerción(25-28).

La comprensión constituye un elemento clave del CI. Aun cuando los individuos hablen el mismo idioma, es frecuente que existan malentendidos en la información que comparten. Por otro lado, en algunas ocasiones los individuos carecen de la información elemental sobre los procedimientos que recibirán y ello obliga a la redacción de documentos extensos, así como al desarrollo de entrevistas reiteradas (el investigador debe ser un excelente comunicador social). Idealmente, el sujeto 
debería tomarse el tiempo que considere oportuno para acceder a ser incluido en un proyecto de investigación(19).

En algunas zonas geográficas, esencialmente del Tercer Mundo, en las cuales, por motivos socioculturales, la colectividad, los esposos e incluso los líderes de grupos son los "responsables" de las decisiones individuales, los investigadores deberían establecer contactos educativos con esas personas y, más aún, lograr empatía, confianza y respeto(19).

El CI no exige que el profesional se instale en una posición pasiva para evitar "interferir" en la libertad del sujeto en investigación; al contrario, demanda que no lo abandone y se comprometa con él en el proceso de toma de decisiones, aconsejándole e incluso persuadiéndole en una determinada dirección. Es cierto que en este proceso abierto el paciente puede estimar como mejores las opciones que el profesional considera peores, pero eso no es un mal resultado, al contrario, es de mayor calidad moral que la mera imposición paternalista.

\section{Conclusiones}

El CI plantea un reto para las investigaciones que involucran seres humanos, por lo que su aplicación se ve fuertemente influida por una amplia gama de factores, entre los que se reconocen los objetivos de las investigaciones, los resultados esperados, las características socioeconómicas del grupo bajo investigación, las capacidades comunicativas de los investigadores y las necesidades reales de los sujetos. El respeto a la autonomía del individuo y a la sociedad, y el estricto compromiso con la verdad científica, reflejados en el CI adecuadamente redactado y conciliado, sería el equilibrio ideal en la investigación y la atención en salud.

\section{Referencias}

1. Miller FG, Joffe S. Limits to research risks. J Med Ethics 2009; 35(7): 445-449.

2. Villafranca R. Investigação científica: sociedade e responsabilidade. In: Guilhem D, Queiroz W, Sorokin P, Zicker F. Ética em pesquisa: compartilhando responsabilidades. Brasília: Letras Livres, Editora UnB, 2009.

3. McCoy M. Autonomy, consent, and medical paternalism: legal issues in medical intervention. $J$ Altern Complement Med 2008; 14(6): 785-792.

4. Ocampo-Martínez J. La bioética y la relación médico-paciente. Cir Ciruj 2002; 70: 55- 59.

5. González-Menéndez R. La relación médico-paciente y su significado en la formación profesional. Educ Med Sup 2003; 17(4).

6. Oliva JE, Bosch C, Carballo R, Fernández-Britto JE. El consentimiento informado, una necesidad de la investigación clínica en seres humanos. Rev Cubana Invest Biomed 2001; 20(2):150-158.

7. Humayun A, Fatima N, Naqqash S, Hussain S, Rasheed A, Imtiaz H, Imam SZ. Patients' perception and actual practice of informed consent, privacy and confidentiality in general medical outpatient departments of two tertiary care hospitals of Lahore. BMC Medical Ethics 2008; 9(14). Disponible en: http://www.biomedcentral.com/content/pdf/1472-6939-9-14.pdf. Acceso el 24 de agosto de 2009.

8. Chan HM. Informed consent Hong Kong style: an instance of moderate familism. J Med Philos 2004; 29: 195-206.

9. Liseckiene I, Liubarskiene Z, Jacobsen R, Valius L, Norup M. Do family practitioners in Lithuania inform their patients about adverse effects of common medications? J Med Ethics 2008; 34: 137-140.

10. Yousaf RM, Fauzi ARM, How SH, Rasool AG, Rohana K. Awareness, knowledge and attitude towards informed consent among doctors in two different cultures in Asia: a cross sectional com- 
parative study in Malaysia and Kashmir, India. Singapore Med J 2007; 48: 559-565.

11. Lower GM. Recordando al maestro Van Rensselaer Potter. En: Acosta Sariego JR, editor. Bioética para la sustentabilidad. La Habana: Publicaciones Acuario, Centro Félix Varela; 1992: 4-6.

12. Annas GJ. Globalized clinical trials and informed consent. N Engl J Med 2009; 360(20): 20502053.

13. Mfutso-Bengo J, Masiye F, Muula A. Ethical challenges in conducting research in humanitarian crisis situations. Malawi Med J 2008; 20(2): 46-49.

14. Sass HM. Reichsrundschreiben 1931: Pre-Nuremberg German Regulations concerning New Therapy and Human Experimentation. The Journal of Medicine and Philosophy 1983; 8: 99-111.

15. Kottow ML. Investigación en seres humanos. Principios éticos internacionales. Cuadernos del Programa Regional de Bioética 1996; 3: 41-52.

16. Katz J. The consent principle of the Nuremberg Code. En: Jonsen A. The birth of bioethics. New York: Oxford University Press;1998: 125-165.

17. The Belmont Report. Ethical Principles and Guidelines for the protection of human subjects of research. The National Commission for the Protection of Human Subjects of Biomedical and Behavioral Research, April 18, 1979. Disponible en http://ohsr.od.nih.gov/guidelines/belmont.html

18. Ad Hoc Committee on Medical Ethics. American College of Physician ethics Manual. Ann Intern Med 1984; 101; 129-137.

19. Marshall PA. Ethical challenges in study design and informed consent for health research in resourcepoor settings. TDR/SDR/SEB/ST/07.1. WHO, Serial Topics No 5.

20. Eriksson S, Höglund AT, Helgesson G. Do ethical guidelines give guidance? A critical examination of eight ethics regulations. Camb Q Healthc Ethics 2008; 17(1): 15-29.

21. Goering S. Postnatal reproductive autonomy: promoting relational autonomy and self-trust in new parents. Bioethics 2009; 23(1): 9-19.

22. Schwartz PH, Meslin EM. The ethics of information: absolute risk reduction and patient understanding of screening. J Gen Intern Med 2008; 23(6): 867-870.

23. Carvalho J, Dias E. Bioética e consentimento informado: revendo a proteção aos vulneráveis. Brasília: Tese Mestrado, Faculdade de Ciências da Saúde, Universidade de Brasília; 2005: 126 f.

24. Bedrossian E, Fernández R. El Consentimiento Informado. Algunas reflexiones para compartir. Rev FASGO 2001; 1(1): 14.

25. Islas-Saucillo M, Muñoz CH. El consentimiento informado. Aspectos bioéticos. Rev Med Hosp Gen Mex 2000; 63(4): 267-273.

26. Brody BA. The ethics of biomedical research: an international perspective. New York: Oxford University Press, 1998.

27. Dawson L, Kass NE. Views of the US researchers about informed consent in international collaborative research. Social Science \& Medicine 2005; 61: 1211- 1222.

28. Woodsong C, Karim QA. A model designed to enhance informed consent: experiences from the HIV Prevention Trials Network. American Journal of Public Health 2005; 95: 412-419.

Recibido: 12 de abril de 2010

Aceptado: 17 de agosto de 2010 